\title{
Quantification of Coronary Inflammation Using Fat Attenuation Index (FAI) in Indians: A Retrospective Study
}

\author{
Atul Kapoor, Aprajita Kapoor, Goldaa Mahajan \\ Advanced Diagnostics and Institute of Imaging, Amritsar, Punjab, India \\ Email address: \\ masatulak@aim.com (A. Kapoor) \\ To cite this article: \\ Atul Kapoor, Aprajita Kapoor, Goldaa Mahajan. Quantification of Coronary Inflammation Using Fat Attenuation Index (Fai) in Indians: A \\ Retrospective Study. Cardiology and Cardiovascular Research. Vol. 5, No. 3, 2021, pp. 129-134. doi: 10.11648/j.ccr.20210503.11
}

Received: June 9, 2021; Accepted: June 23, 2021; Published: June 30, 2021

\begin{abstract}
Background: Asian Indians have the highest prevalence (11\%) of coronary artery risk worldwide compared to all other races. So far no quantifiable risk factor has been shown to explain such a high prevalence. Fat attenuation index (FAI) using CT coronary angiography (CTCA) has been recently used to demonstrate coronary perivascular inflammation and to confirm that atherosclerosis is an inflammatory process. Hence this study was conducted to determine FAI using CTCA in 200 adult Indians as a retrospective study to determine if it can be the answer to establish the cause for high prevalence of CAD in Indians and whether there are differences in FAI in normal adults and those with significant CAD and to determine if it could be used as imaging biomarker for diagnosis and follow up of such patients. Material and Methods: Retrospective study of 200 patients who underwent CTCA was done. Patients were divided into two groups based on no coronary disease (NOCAD) and those with significant coronary artery disease (CAD). Patient demographics were recorded for both groups. FAI estimation was done along with Plaque volume index (PVI), pericardial volume estimation and the differences between the two groups were statistically analysed. Results: Mean patient age in both groups was 52 years with male predominance (75-80\%). Mean FAI,s for left anterior descending and right coronary arteries was 45.4 and $-44.7 \mathrm{HU}$ and of -38.0 and $-39.2 \mathrm{HU}$ for NOCAD and CAD groups respectively $(\mathrm{p}<0.001)$. Sensitivity and specificity of FAI to differentiate NOCAD from CAD at a cut off value >$38 \mathrm{HU}$ was $73 \%$ and $80 \%$ respectively with LR of 3.6. Conclusion: Normal adult Indians with NOCAD showed a high FAI compared to all other races which could be reason for highest prevalence of CAD amongst Indians. FAI can be used as imaging biomarker to differentiate CAD from NOCAD with sensitivity and specificity of $73 \%$ and $80 \%$ respectively.
\end{abstract}

Keywords: Coronary Artery Disease, CT Coronary Angiography, Fat Attenuation Index

\section{Introduction}

The prevalence of coronary artery disease (CAD) in the US is $2.5 \%$ [1]. The prevalence of CAD risks in Indians living in India is $11 \%$ for non-diabetic patients and $21.4 \%$ for diabetic patients [2]. Asian Indians stand out in that their $\mathrm{CAD}$ rates remain higher even after immigration to US, compared to other ethnic populations and are three times higher than US national average $[3,4]$. This suggests some non-modifiable risk factors for $\mathrm{CAD}$ in the Asian Indian population which needs to be evaluated. Vascular inflammation is a driver of coronary atherosclerotic plaque formation and is a typical feature of atherosclerotic plaque rupture, leading to acute coronary syndrome. Currently no method is readily available to allow early detection of vascular inflammation in coronary arteries. Such a method would enable timely deployment of measures to prevent disease development and future heart attacks. So far coronary CT angiography (CTCA) has focused predominantly on identification of anatomically significant coronary artery stenosis, which is seen in fewer than $50 \%$ of patients referred for this test $[5,6]$. FAI (fat attenuation index) is one such method recently devised using CTCA in which perivascular fat has been developed as a imaging biomarker to detect and measure the changes in the perivascular coronary artery fat as a proof to validate the hypothesis that coronary inflammation results in impaired lipid accumulation and adipocyte differentiation in adjacent perivascular fat thus resulting in reduced fat content in response to inflammatory signals from the adjacent coronary artery providing the proof of concept of a so-called "sensor of coronary inflammation"[7-9]. We conducted a retrospective study and analyzed data of 200 
Indian patients with no previous labelled coronary artery disease who had undergone CTCA to determine a): FAI of coronary arteries in adult Indians with no coronary artery disease. b): To determine the change in FAI between normal adult Indian and those with significant coronary artery disease. c): To estimate if FAI could be the single risk factor leading to high prevalence of CAD in Indians.

\section{Material and Methods}

Study comprised of retrospective analysis of data of 200 patients from the local registry who had undergone CTCA in 2019-2020. Labels and identifiers from entire data were removed. Waiver for informed consent was obtained from local ethics committee. Patient demographics including age, sex, and body mass index, family history of CAD, diabetes, dyslipidemia and hypertension were recorded. Any patient with history of oral intake of statins or disprin or prior proven CAD was excluded from the study.

\subsection{Imaging Protocol}

CT examination was done ona dual energy CT 128 scanner (Siemens Go-Top, Forchheim, Germany.. Oral $50 \mathrm{mg}$ metoprolol one hour before the examination was given followed by sublingual nitroglycerin $0.5 \mathrm{mg}$ to all patients except who had any history of hypotension. A retrospectively gated ECG triggered spiral (pitch 0.3) acquistion was done with collimation of and gantry rotation time of $0.33 \mathrm{~ms}$ at a tube voltage of $70 \mathrm{KV}$ along with automated tube current modulation using (CARE Dose 4D, Siemens healthineers). The quality reference voltage and current was set to $120 \mathrm{kV}$, 320 mAs. R-R interval was fixed at $35-75 \%$. $35 \mathrm{ml}$ of non iodinated contrast iomperol 400 (Iomeron Bracco UK Ltd) was injected using bolus tracking soft ware with dual head injector (MEDRAD, Stellant, Bayers, Munich, Germany) with flow rate of $4 \mathrm{ml} / \mathrm{s}$ followed by $20 \mathrm{ml}$ saline. Image reconstruction was done using iterative reconstruction (SAFIRE, Siemens healthineers level 3) with reconstruction kernel of BV36.

\subsection{Image Analysis}

Siemens Syngo. via workstation was used for post processing in multiplanar and volume rendering projections. Vessels with stenosis more than $50 \%$ was labeled as significant. CTFFR was done onsite on Siemens Syngovia Frontier workstation using CTFFR (version2.0) software. CTFFR was displayed in the form of color maps of coronary tree with blue being normal and red being ischemic vessels and those showing FFR below 0.8 distal to the stenotic site were taken as haemodynamically significant. Plaque analysis was done using same data set with Plaque analysis software version 5.0. Plaque volume index (PVI) was calculated as total plaque burden in $\mathrm{cm} 3 /$ vessel length in $\mathrm{cm} \times 100 \%$. PVI of more than 0.2 was taken as cut off for significant atherosclerosis. High risk plaque features like spotty calcifications, positive remodelling, napkin ring sign were also recorded. Perivascular fat attenuation index with range from -150 to $-20 \mathrm{HU}$ from proximal $4 \mathrm{~cm}$ length of right coronary and left anterior descending coronary arteries. Pericardial volume estimation was then done using Cardiac risk assessment software version 1.2.2 and a value of more than $100 \mathrm{ml}$ was taken as abnormal.

\subsection{Statistical Analysis}

Was done using Analyse-IT software. Means of all continuous variables were compared using student's $t$ test with $\mathrm{p}$ value of more than 0.05 being statistically insignificant. Sensitivity and specificity was determined for diagnostic performance along with likelihood ratio with area under curve to determine the best cut off value for predictability of significant CAD.

\section{Results}

The study comprised of 200 patients in two groups of 100 patients each with those having no significant coronary artery disease (NOCAD) and those with significant coronary artery disease (CAD). Patient demographics are shown in [Table 1]. Mean age of patients was 52 years in both the groups with 80 and 75 being males in group NOCAD and CAD groups respectively. BMI was high in both the groups with similar other patient demographics as enlisted in table 1. Table 2 shows the imaging characteristics of both the groups. The mean Fat attenuation index (FAI) in NOCAD group was 45.4 and $-44.7 \mathrm{HU}$ in left anterior descending artery and right coronary artery respectively [Figures 1-5]. CAD group showed mean FAI of -38.0 and $-39.2 \mathrm{HU}$ in left anterior descending and right coronary artery, differences being statistically significant $(\mathrm{p}<0.001)$ [Figures 6-12]. Statistically significant differences were also seen in pericardial volume and PVI in both the groups with means of $131 \mathrm{ml}$ and $151 \mathrm{ml}$ and 0.25 and 0.34 respectively in NOCAD and CAD groups. CACS was also higher in group CAD with mean score of 25.4 [Table 2]. The Sensitivity and specificity of FAI in determining the presence of significant CAD at a cut off score of more than -38.0 was $73 \%$ and $80 \%$ respectively with likelihood ratio of 3.6 in the present study [figure 13].

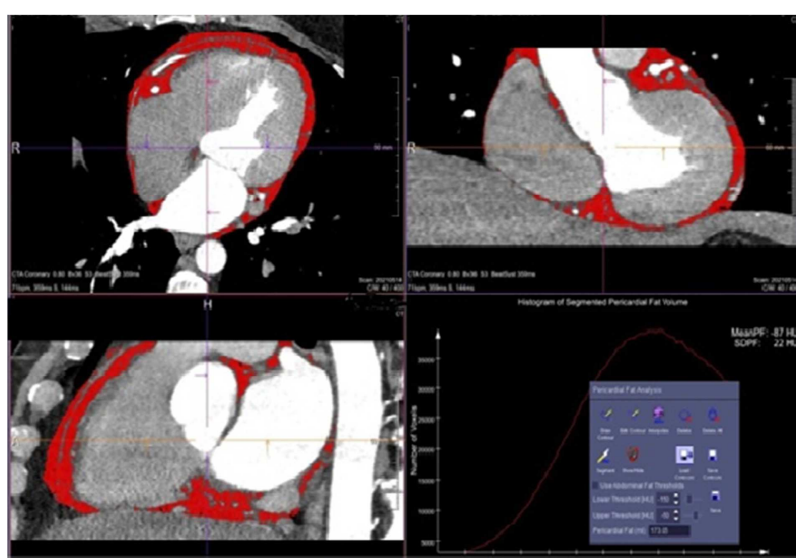

Figure 1. NOCAD patient with increased pericardial fat volume $173 \mathrm{ml}$. 


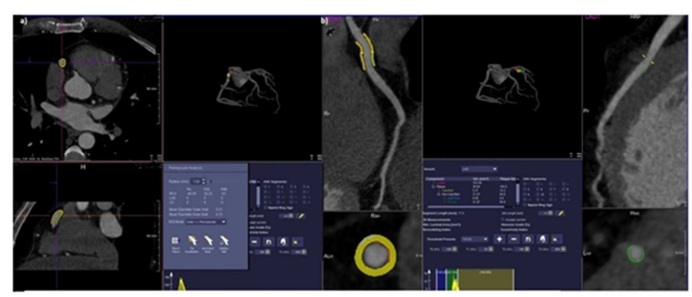

Figure 2. a) CTCA showing FAI of $-46.5 H U$ in right coronary artery. b) Plaque analysis showing fibrotic plaque with PVI of 0.17.

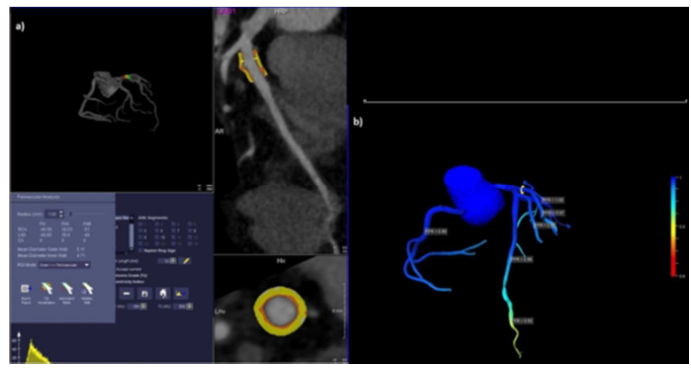

Figure 3. a) CTCA showing FAI of -40.65 in proximal left anterior descending artery b) CTFFR showing no ischemia.

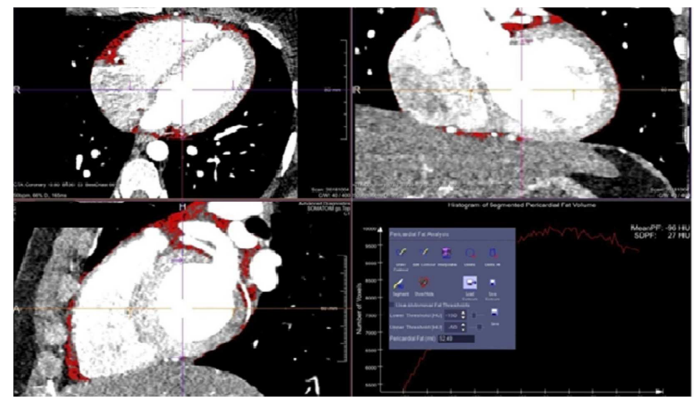

Figure 4. Pericardial fat volume estimation in NOCAD patient with normal pericardial fat volume of $52.49 \mathrm{ml}$.

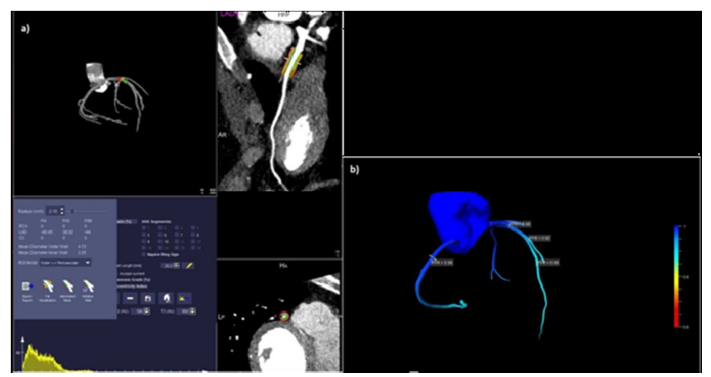

Figure 5. a) CTCA showing FAI of -40.5 in proximal left anterior descending artery. b) Normal CTFFR with no ischemia.

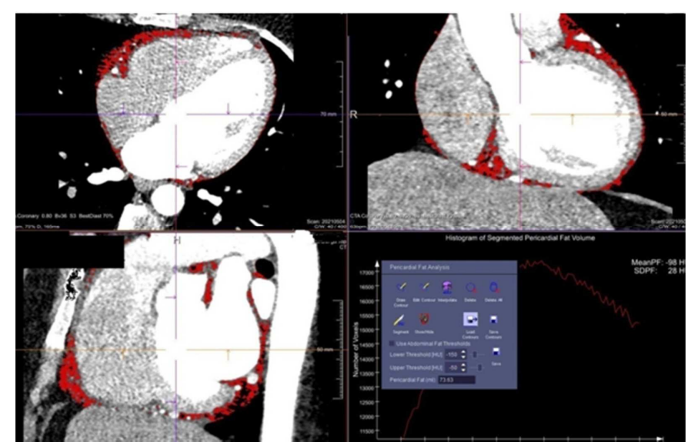

Figure 6. Pericardial fat volume of $73.6 \mathrm{ml}$ in a patient with CAD.

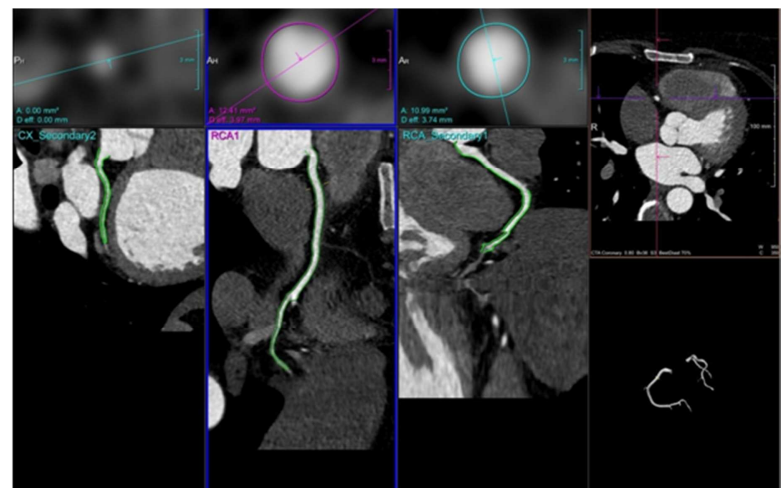

Figure 7. CTCA showing normal coronary arteries with no anatomic stenosis.

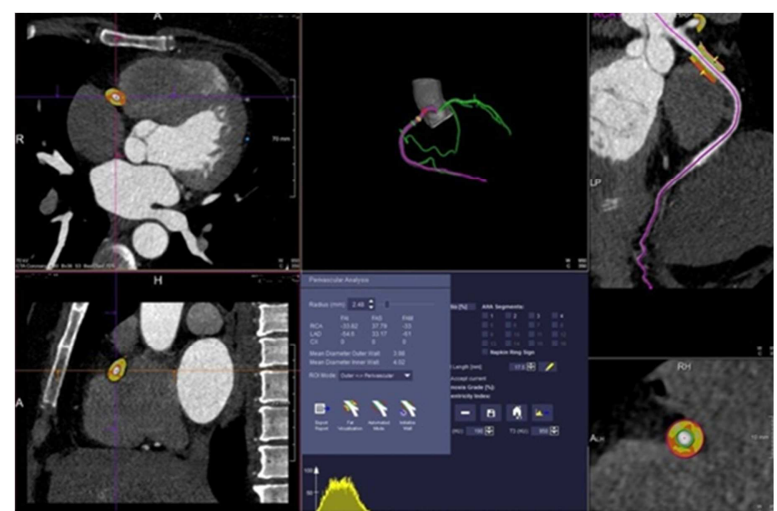

Figure 8. CTCA showing FAI of -33.8 HU in right coronary artery and -54.2 in left anterior descending artery.

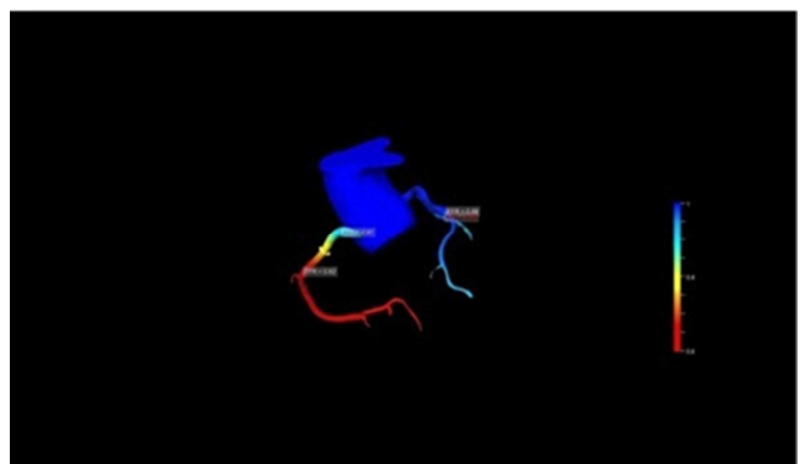

Figure 9. CTFFR of same patient showing reduced FFR of 0.52 in mid and distal right coronary artery- INOCA.

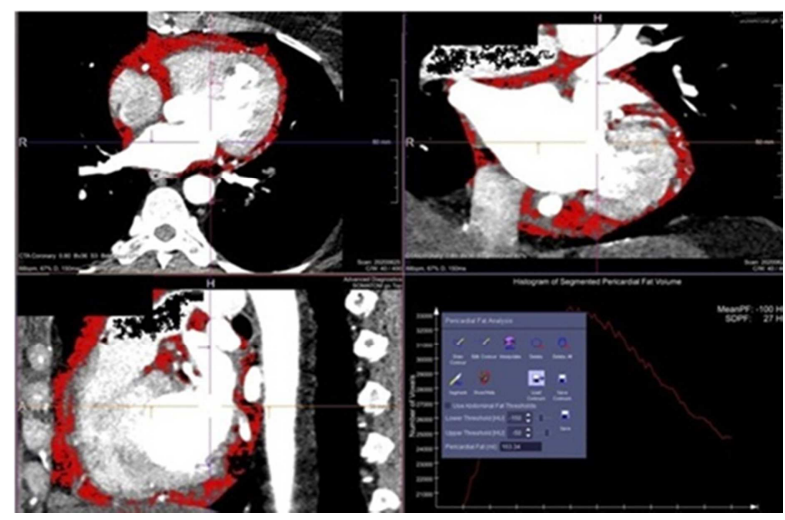

Figure 10. Pericardial fat volume estimation of $163.4 \mathrm{ml}$ in CAD patient. 


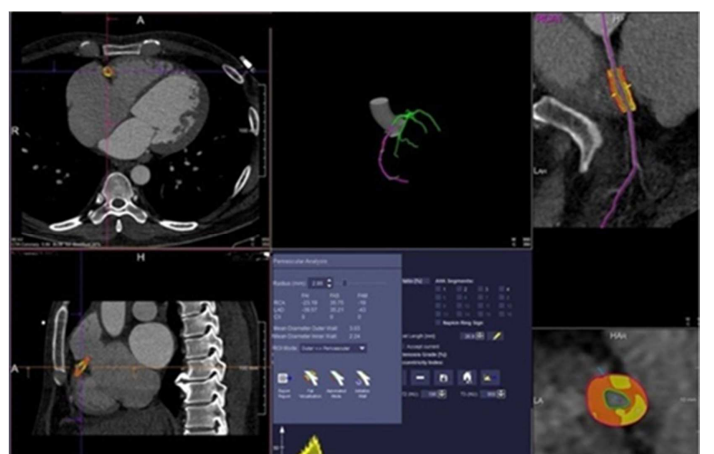

Figure 11. CTCA showing FAI of $-23.9 \mathrm{HU}$ and $-39.5 \mathrm{HU}$ in the same patient with $C A D$

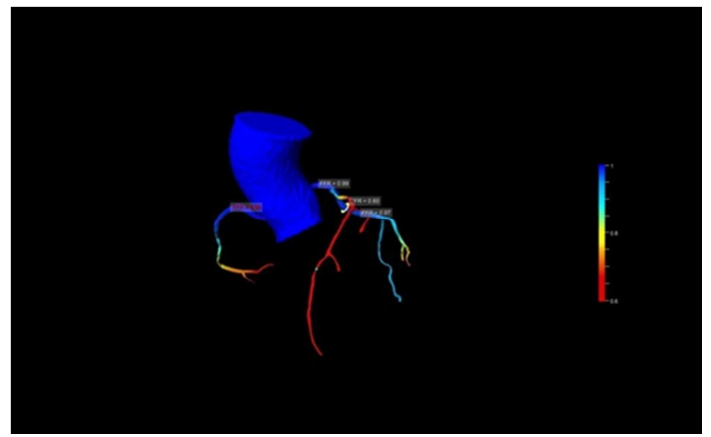

Figure 12. CTFFR showing obstructive ischemia in left anterior descending artery with distal macrovascular endothelial dysfunction in right coronary artery.

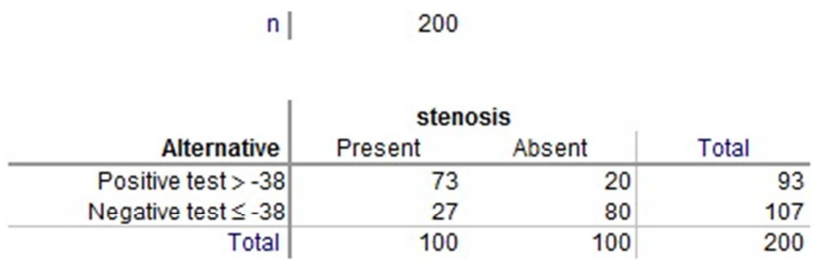

Sample prevalence | $\quad 0.498$

\begin{tabular}{r|r|c} 
& & $95 \% \mathrm{Cl}$ \\
\hline Sensitivity - TP proportion & 0.730 & 0.632 to 0.814 \\
Specificity - TN proportion & 0.802 & 0.711 to 0.875 \\
FP proportion & 0.198 & 0.125 to 0.289 \\
FN proportion & 0.270 & 0.186 to 0.368 \\
& & \\
Likelihood ratio (+) & 3.69 & \\
Likelihood ratio (-) & 0.34 &
\end{tabular}

At sample prevalence

\begin{tabular}{r|r|r|}
$\begin{array}{r}\text { Correct classification } \\
\text { Misclassification }\end{array}$ & 0.766 & 0.701 to 0.823 \\
& 0.234 & 0.177 to 0.299 \\
& & \\
Positive predictive value & 0.785 & 0.688 to 0.863 \\
egative predictive value & 0.750 & 0.657 to 0.828
\end{tabular}

Figure 13. Diagnostic performance of FAI to detect CAD on CTCA.

Table 1. Patient Demographics.

\begin{tabular}{lllll}
\hline Sno & Parameters & Group I (NOCAD) $\mathbf{n = 1 0 0}$ & Group II (CAD) n=100 & P Value \\
\hline 1. & Mean Age & 52 & 52 & 0.83 \\
2. & Sex & & & \\
& Males & 80 & 75 & 0.6 \\
& Females & 20 & 25 & 0.11 \\
3. & BMI & 28.2 & 29.2 & 0.15 \\
4. & Hypertension & 74 & 91 & 0.22 \\
5. & Diabetes & 38 & 41 & 0.11 \\
6. & Smoking & 7 & 9 & X \\
7. & Dyslipidemia & 51 & 68 & 0.13 \\
8. & Family H/o CAD & 58 & 65 & 0.11 \\
9. & Sedentry Lifestyle & 78 & 85 & 0.13 \\
\hline
\end{tabular}

Table 2. Imaging Characteristics of NOCAD and CAD groups.

\begin{tabular}{|c|c|c|c|c|}
\hline Sno. & Parameters & Group I NOCAD & Group II CAD & p Value \\
\hline 1. & Mean Plaque Volume Index (PVI) & 0.25 & 0.34 & 0.002 \\
\hline 2. & Mean Pericardial Volume (PV) $\mathrm{ml}$ & 131 & 151 & 0.04 \\
\hline 3. & Coronary calcium Score (CACS) & 0.9 & 25.4 & $<0.001$ \\
\hline 4. & Number of Percentage Stenosis $>50 \%$ & 0 & 60 & $<0.001$ \\
\hline \multirow[t]{2}{*}{5 . } & Perivasc. Fat attenuation index (FAI) Left anterior descending artery & -45.4 & -37.9 & $<0.001$ \\
\hline & Right coronary artery & -44.7 & -39.2 & $<0.001$ \\
\hline
\end{tabular}

Table 3. Comparison of FAI amongst racially different normal Adult males.

\begin{tabular}{llll}
\hline \multirow{2}{*}{ Sno } & \multirow{2}{*}{ RACE } & FAI (HU) & \\
\cline { 3 - 4 } & & LAD & RCA \\
\hline 1 & Japanese (4) & -71.46 & -72.2 \\
$2 \mathrm{a}$ & European1 (5) & -92.4 & -88.4 \\
$2 \mathrm{~b}$ & European 2 (10) & -78 & -75.1 \\
3 & American (10) & -77 & -75 \\
4 & Indian & -45 & -44 \\
\hline
\end{tabular}

\section{Discussion}

CAD risk in Indians is reported to be significant higher in the general population than in Caucasians [9]. Our retrospective study quantified the $\mathrm{CAD}$ risk in the form of high FAI in normal adult Indians in both left anterior descending and right coronary of coronary arteries with a mean of -44.5 and $-45.0 \mathrm{HU}$ respectively. This was much 
higher than the cut off of -70 HU shown in CRISP trial [10] in which FAI was used to predict clinical outcomes in 4000 individuals undergoing CCTA as part of their clinical care in Erlangen, Germany and Cleveland, USA (as derivation and validation cohorts respectively, with -2000 individuals each). CRISP trial [10] showed that individuals with perivascular FAI $>-70.1 \mathrm{HU}$, the risk for all-cause mortality had $2.55 \mathrm{HR}$ for the derivation and 3.69 for the validation cohort while the normal adults in the study showed a mean -75.1 HU in derivation cohort and $-77.0 \mathrm{HU}$ in validation cohort which was much lower than the mean attenuation of $-44.5 \mathrm{HU}$ and $44 \mathrm{HU}$ seen in the present study. Using the same cut off value of $-70 \mathrm{HU}$ suggested in CRISP trial [10] our results suggest a much higher risk of future coronary events in both normal adult Indians and those with significant CAD which explains the high endemic burden of CAD amongst Indians. We compared the results of the present study with other studies using FAI [Table 3] which clearly reveal that normal Indian adults have the highest mean FAI thereby high coronary inflammation as compared to the studies done on other races. Perivascular coronary fat is a source of multiple bioactive cytokines such as leptin, adiponectin, resistin, plasminogen activator inhibitor-1, apelin, tumour necrosis factor-alpha (TNF- $\alpha$ ), interleukin-6 and monocyte chemoattractant protein-1 (MCP-1), which are involved in the regulation of endothelial function, coagulation and inflammation through paracrine and endocrine actions and plasma inflammatory biomarkers like HsCRP may not adequately reflect this local tissue inflammation $[11,12]$. It is postulated that in the adipose tissue, hypoxia leads to increased expression of inflammatory genes and decreased expression of adiponectin [13]. Sacks et al [12] in their study suggested that there is upregulation and higher expression of pro-inflammatory chemokinesMCP-1, TNF- $\alpha$ and VCAM-1 in the perivascular epicardialfat with reduced expression of anti-inflammatory chemokines, adiponectin, ADORA-1 and UCP-1 which are cardioprotective. MCP-1 initiates macrophage infiltration of adipose tissue, a hallmark of many studies which have reported presence of inflammatory state of EAT in CAD patients [14, 15-17]. Hirata et al [14] have reported the presence of greater number of M1 macrophages (inflammatory) as compared to M2 (inactive) in epicardial fat in CAD patients. All these changes lead to conversion of perivascular fat into aqueous phase thus resulting in higher FAI. The fact that atherosclerotic lesions develop in those parts of coronary arteries, which are surrounded completely by epicardial fat and the amount of fat and macrophage infiltration correlate with atherosclerotic plaque size and composition, further emphasizes the inflammatory nature of disease. Our study shows that normal adult Indians have active subclinical perivascular inflammation resulting in higher FAI. Persistent longtime subclinical upregulation of inflammatory markers and down regulation of adiponectin leads to local plaque formation, stenosis and rupture. In the present study patients with significant CAD had higher FAI of more than -40 and using a cut off of FAI higher -38 had a sensitivity and specificity of $73 \%$ and $78 \%$ with likelihood ratio of 3.6 in the detection of significant CAD. This could be an important factor in not only diagnosis but also prognosis of CAD patients. A patient with INOCA in the present study (shown in figures 6-9) had markedly reduced FAI in the culprit vessel which had ischemia but with no anatomical stenosis. Yu et al [18] have recently shown that FAI along with plaque volume estimation correlated well with degree of stenosis and ischemia. Similarly Kluneretal [19] also opined that use of FAI has significant prognostic value in both primary and secondary prevention of CAD and offers a significant improvement in cardiac risk discrimination beyond traditional risk factors. Similar results have been shown in the current study where conventional coronary risk factors did not show any statistical significant difference in the NOCAD and CAD groups $\mathrm{s}$ however statistically significant differences were seen in CACS and Plaque volume index between the two groups. This suggests that FAI can be useful adjunctive biomarkers in treatment planning and follow up of such patients. Pericardial fat volume was higher as was BMI in both the groups in the present study and had no discriminative value. The limitation seen in the current study was that it was retrospective and no follow up of the patients was not done to assess outcome and prevalence of major cardiac events in either of the groups.

\section{Conclusion}

The study concludes that FAI estimation by CTCA shows a high prevalence of coronary inflammation in clinically normal Indian adults with mean FAI of -44 and $45 \mathrm{HU}$ and was a single statistically significant imaging biomarker for detection of CAD and is the likely reason for high prevalence of CAD in Asian Indians. FAI was also seen as a better biomarker than all traditional risk factors for patients with both subclinical and symptomatic CAD.

FAI had a sensitivity of $73 \%$ and specificity of $80 \%$ with likelihood ratio of 3.6. in the present study to differentiate patients with subclinical coronary inflammation in normal adults from those with significant $\mathrm{CAD}$ at a cut off value of $>-38.0$.

\section{Acknowledgements}

Reshmi Mukheerji, Application Support Asia Pacific Siemens Healthineers.

\section{References}

[1] Ross R. Atherosclerosis: an inflammatory disease. $N$ Engl J Med. 1999; 340: 115-126.

[2] Mohan V, Deepa R, Rani SS, et al. Prevalence of coronary artery disease and its relationship to lipids in a selected population in South India: The Chennai Urban Population Study (CUPS No. 5). J Am CollCardiol 2001; 38: 682-7. 10.1016/S0735-1097(01)01415-2. 
[3] Enas EA, Senthilkumar A. Coronary artery disease in Asian Indians: an update and review. Internet J Cardiol 2001; 1.

[4] Sharma M, Ganguly NK. Premature coronary artery disease in Indians and its associated risk factors. Vasc Health Risk Manag 2005; 1: 217-25.

[5] Douglas PS, Hoffmann U, Patel MR. Outcomes of anatomical versus functional testing for coronary artery disease. $N$ Engl $J$ Med. 2015; 372: 1291-1300.

[6] SCOT-HEART investigators CT coronary angiography in patients with suspected angina due to coronary heart disease (SCOT-HEART): an open-label, parallel-group, multicentre trial. Lancet. 2015; 385: 2383-2391.

[7] Antonopoulos AS, Sanna F, Sabharwal N. Detecting human coronary inflammation by imaging perivascular fat. SciTransl Med. 2017; 9: eaal2658.

[8] T. Sugiyama, Y. Kanaji, M. Hoshino, M. Yamaguchi, M. Hada, T. Misawa, Y. Sumino, K. Nogami, H. Ueno, T. Kakuta. Prognostic value of fat attenuation index of pericoronary adipose tissue surrounding left anterior descending artery on coronary computed tomography angiography. European Heart Journal, Volume 41, Issue Supplement_2, November 2020, ehaa 946.1346.

[9] Ma R, Ties D, Assen MV, Pelgrim GJ, Sidorenkov G, van Ooijen PM etal. Towards reference values of pericoronary adipose tissue attenuation: impact of coronary artery and tube voltage in coronary computed tomography angiography. European Radiology (2020) 30: 6838-6846.

[10] Oikonomou E. K., Marwan M., Desai M. Y. Non-invasive detection of coronary inflammation using computed tomography and prediction of residual cardiovascular risk (the CRISP CT study): a post-hoc analysis of prospective outcome data. Lancet (London, England) 2018; 392: 929-939.

[11] Baker AR, Silva NF, Quinn DW, Harte AL, Pagano D, Bonser RS, et al. Human epicardial adipose tissue expressesa pathogenic profile of adipocytokines in patients withcardiovascular disease. Cardiovasc Diabetol 2006; 5: 1.
[12] Sacks HS, Fain JN, Cheema P, Bahouth SW, Garrett E, Wolf $\mathrm{RY}$, et al. Inflammatory genes in epicardial fat contiguouswith coronary atherosclerosis in the metabolic syndromeand type 2 diabetes: Changes associated with pioglitazone. Diabetes Care 2011; 34: 730-3.

[13] Lacobellis G, Bianco AC. Epicardial adipose tissue: Emergingphysiological, pathophysiological and clinical features. Trends Endocrinol Metab 2011; 22: 450-7.

[14] Mazurek T, Zhang L, Zalewski A, Mannion JD, Diehl JT, Arafat $\mathrm{H}$, et al. Human epicardial adipose tissue is a sourceof inflammatory mediators. Circulation 2003; 108: 2460-6.

[15] Hirata Y, Tabata M, Kurobe H, Motoki T, Akaike M, Nishio $\mathrm{C}$, et al. Coronary atherosclerosis is associated with macrophagepolarization in epicardial adipose tissue. J Am Coll Cardiol 2011; 58: 248-55.

[16] Xu H, Barnes GT, Yang Q, Tan G, Yang D, Chou CJ, et al. Chronic inflammation in fat plays a crucial role in the development of obesity-related insulin resistance. J ClinInvest 2003; 112: 1821-30.

[17] Weisberg SP, McCann D, Desai M, Rosenbaum M, Leibel RL, Ferrante AW Jr. Obesity is associated withmacrophage accumulation in adipose tissue. J ClinInvest 2003; 112: 1796808 .

[18] Yu M, Dai X, Deng J, Lu Z, Shen C, Zhang J. Diagnostic performance of perivascular fat attenuation index to predict hemodynamic significance of coronary stenosis: a preliminary coronary computed tomography angiography study. Eur Radiol. 2020 Feb; 30 (2): 673-681.

[19] Kluner LV, Oikonomu EK, Phil D, Antoniade C. Assessing Cardiovascular Risk by Using the Fat Attenuation Index in Coronary CT Angiography. Radiology: Cardiothoracic Imaging 2021; 3 (1): 200563 https://doi.org/10.1148/ryct.2021200563. 